\title{
CORRIGENDUM
}

\section{Effects of a high-fat-high-fructose diet, stress and cinnamon on central expression of genes related to immune system, hypothalamic-pituitary- adrenocortical axis function and cerebral plasticity in rats - CORRIGENDUM}

\author{
Nathalie Marissal-Arvy, Cécile Batandier, Julien Dallennes, Frédéric Canini, Laurent Poulet, \\ Karine Couturier, Isabelle Hininger-Favier, Marie-Pierre Moisan, Anne-Marie Roussel and \\ Pierre Mormède
}

(First published online 14 February 2014)

doi:10.1017/S0007114513003577, Published by Cambridge University Press, 20 November 2013.

The authors have indicated that their conclusion that cinnamon could be 'deleterious under the unbalanced diet encountered in Western societies ${ }^{(1)}$ is not supported by the findings of the study and have requested this conclusion be removed from the abstract of the article. The Editors of the BrJ Nutr agreed with the author's request. The revised abstract is given below.

\begin{abstract}
The intake of a high-fat/high-fructose ( $\mathrm{HF} / \mathrm{HFr}$ ) diet is described to be deleterious to cognitive performances, possibly via the induction of inflammatory factors. An excess of glucocorticoids is also known to exert negative effects on cerebral plasticity. In the present study, we assessed the effects of an unbalanced diet on circulating and central markers of inflammation and glucocorticoid activity, as well as their reversal by dietary cinnamon (CN) supplementation. A group of male Wistar rats were subjected to an immune challenge with acute lipopolysaccharide under a HF/HFr or a standard diet. Another group of Wistar rats were fed either a HF/HFr or a control diet for 12 weeks, with or without CN supplementation, and with or without restraint stress (Str) application before being killed. We evaluated the effects of such regimens on inflammation parameters in the periphery and brain and on the expression of actors of brain plasticity. To assess hypothalamic-pituitary-adrenocortical axis activity, we measured the plasma concentrations of corticosterone and the expression of central corticotrophin-releasing hormone, mineralocorticoid receptor, glucocorticoid receptor and 11ß-hydroxysteroid dehydrogenase. We found that the HF/HFr diet induced the expression of cytokines in the brain, but only after an immune challenge. Furthermore, we observed the negative effects of Str on the plasma concentrations of corticosterone and neuroplasticity markers in rats fed the control diet but not in those fed the $\mathrm{HF} / \mathrm{HFr}$ diet. Additionally, we found that $\mathrm{CN}$ supplementation exerted beneficial effects under the control diet, but that its effects were blunted or even reversed under the HF/HFr diet. CN supplementation could be beneficial under a standard diet.
\end{abstract}

\section{Reference}

1. Marissal-Arvy N, Batandier C, Dallennes J, et al. Effect of a high-fat-high-fructose diet, stress and cinnamon on central expression of genes related to immune system, hypothalamic-pituitary-adrenocortical axis function and cerebral plasticity in rats. Br J Nutr. Published by Cambridge University Press, 20 November 2013, doi:10.1017/S0007114513003577. 